\title{
Tumor $\mathrm{pO}_{2}$ as a surrogate marker to identify therapeutic window during metronomic chemotherapy of $9 \mathrm{~L}$ gliomas
}

\author{
Sriram Mupparaju ${ }^{1,2}$, Huagang Hou ${ }^{1,2}$, Jean P. Lariviere ${ }^{1}$, Harold M Swartz ${ }^{1,2}$, and Nadeem \\ Khan ${ }^{1,2}$ \\ ${ }^{1}$ EPR Center for Viable Systems, Dartmouth Medical School, Hanover, NH 03755 \\ ${ }^{2}$ Norris Cotton Cancer Center, Dartmouth-Hitchcock Medical Center, Lebanon, NH 03756
}

\section{Abstract}

Glioblastomas are aggressive and highly vascularized primary brain tumors with a 5-year survival rate of less than $10 \%$. Anti-angiogenic approaches are being investigated for potential therapeutic benefits for this fatal malignancy. However, lack of suitable markers that can be used to monitor therapeutic effects during such treatments has restricted their optimization. We have focused on the development of tumor $\mathrm{pO}_{2}$ as a surrogate marker to identify therapeutic window during antiangiogenic approaches, such as metronomic chemotherapy. We report the effect of four weekly administrations of cyclophosphamide $\left(140 \mathrm{mg} / \mathrm{Kg}\right.$, i.p), a chemo drug, on tumor $\mathrm{pO}_{2}$ and growth of subcutaneous 9L tumors in SCID mice. The repeated measurement of tumor $\mathrm{pO}_{2}$ was carried out using in vivo EPR oximetry. The subcutaneous $9 \mathrm{~L}$ tumors were hypoxic with a pre-treatment tumor $\mathrm{pO}_{2}$ of $5.1 \pm 1 \mathrm{mmHg}$ and a tumor volume of $236 \pm 45 \mathrm{~mm}^{3}$ on day 0 . The tumor $\mathrm{pO}_{2}$ increased significantly to $26.2 \pm 2 \mathrm{mmHg}$ on day 10 , and remained at an elevated level till day 31 during weekly treatments with cyclophosphamide. The tumor $\mathrm{pO}_{2}$ then declined to $20 \pm 9 \mathrm{mmHg}$ on day 43. The tumor volume of the control group increased significantly with no change in tumor $\mathrm{pO}_{2}$ over days.

Results indicate a transient increase in tumor $\mathrm{pO}_{2}$ during metronomic chemotherapy of $9 \mathrm{~L}$ gliomas and could be potentially used as a marker to identify vessel normalization during metronomic chemotherapy. The ability to identify therapeutic window non-invasively using EPR oximetry could have a significant impact on the optimization of clinical protocols. In vivo EPR oximetry is currently being tested for repeated $\mathrm{pO}_{2}$ measurements in patients with superficial tumors.

\section{Introduction}

Gliomas are malignant brain tumors with a poor prognosis. The median survival is less than one year from the time of diagnosis and even in most favorable situations; most patients do not survive beyond two years despite aggressive treatment protocols [1-3]. Multimodality approaches with systemic chemotherapy administered concurrently and as an adjuvant with radiotherapy have only shown modest survival advantages and are usually accompanied with acute or late toxicity.

New therapeutic approaches, based on tumor genetics or physiology, are urgently needed that can improve the outcome for patients with this highly malignant tumor. However, lack of appropriate markers that can guide these approaches to enhance their effect has restricted their optimization. The approach of metronomic chemotherapy, which is aimed at preferentially targeting tumor vasculature to achieve an anti-angiogenic effect, has shown 
low toxicity and some advantages in pre-clinical trials $[4,5]$. However, the effect of metronomic regimens is likely to vary with the dose and the interval between doses and therefore may significantly alter the therapeutic outcome. Unfortunately, the currently used read-outs, namely, time to progression, progression free survival, and median survival, can be assessed only at the end of the treatment and are not suitable markers to guide dose optimization, identify non-responders, and assess efficacy during treatment. Therefore, reliable markers that are indicative of the efficacy and can identify non-responders early during the course of therapy are vital to achieve the best therapeutic results.

We hypothesize that metronomic chemotherapy will lead to an increase in tumor $\mathrm{pO}_{2}$ (partial pressure of oxygen) associated with changes in the tumor vasculature (antiangiogenic effect). These changes in tumor $\mathrm{pO}_{2}$ could be used as a potential surrogate marker to optimize dose regimens, identify non-responders, and predict outcome. We report the results of our ongoing study on the effect of metronomic chemotherapy using 4 weekly treatments with cyclophosphamide (CPA) on tissue $\mathrm{pO}_{2}$ and growth of subcutaneous 9L gliomas in SCID mice.

\section{Methods}

\subsection{Animal and tumor models}

All animal-use procedures were in strict accordance with the NIH Guide for the Care and Use of Laboratory Animals and were approved by the Institutional Animal Care and Use Committee of Dartmouth Medical School. The 9L cells were grown in vitro in DMEM medium with $10 \%$ FBS and $1 \%$ penicillin-streptomycin. For injection, the cells were trypsinized and suspended in DMEM, without serum or additives. The tumors of $6-8 \mathrm{~mm}$ in length were obtained in $14-16$ days by subcutaneous injection of $100 \mu$ l cell suspension $\left(4 \times 10^{5}\right.$ cells $)$ with a 25 -gauge needle in the left flank of anesthetized $(2-2.5 \%$ isoflurane with $30 \% \mathrm{O}_{2}$ ) SCID mice (Charles River Laboratories, MA).

\subsection{Implantation of oximetry probe (LiPc) for $\mathrm{pO}_{2}$ measurements using multi-site EPR oximetry}

Once the tumors reached a size of $6-8 \mathrm{~mm}$ in length, the mice were anesthetized $(2-2.5 \%$ isoflurane with $30 \% \mathrm{O}_{2}$ ), and two aggregates of LiPc crystals ( $40-50 \mu \mathrm{g}$ each) were injected into the tumors ( $4 \mathrm{~mm}$ apart, $2 \mathrm{~mm}$ depth) using 25 gauge needles. Once injected, these LiPc deposits provided repeated tissue $\mathrm{pO}_{2}$ measurements at two sites of each tumor simultaneously using multi-site Electron Paramagnetic Resonance (EPR) oximetry [6-8].

For $\mathrm{pO}_{2}$ measurements, the mice were anesthetized $\left(1.5 \%\right.$ isoflurane, $\left.30 \% \mathrm{O}_{2}\right)$ and positioned between the poles of the EPR magnet. The external loop resonator was placed close to the surface of the tumor and a pre-treatment (baseline) EPR measurements were acquired for 30 minutes on day 0 . The EPR spectra will be averaged for 1 minute each to improve signal to noise ratio. Typical settings for the spectrometer were incident microwave power, $4-6 \mathrm{~mW}$; magnetic field center, 400 gauss; scan range, 2 gauss; modulation frequency, $27 \mathrm{kHz}$. Modulation amplitude was set at less than one-third of the EPR line width. During EPR measurements, the temperature of the animals was monitored using a rectal probe and maintained at $37 \pm 0.5^{\circ} \mathrm{C}$ using a thermostatically controlled heated pad and a flow of warm air. CPA was prepared in phosphate buffered saline (PBS, Mediatech Inc. $\mathrm{VA})$ and the mice received single doses (140 mg/kg, i.p) on days $0,7,14$ and 21 . The tumor $\mathrm{pO}_{2}$ and volume measurements were continued for approximately seven weeks. Similar protocol was used for the control group and the mice were treated with the vehicle (PBS) only. 


\subsection{Tumor volume measurements}

The tumor volumes were measured using the formula: $\pi / 6 \times$ length $\times$ width $^{2}$. This is a well established procedure for tumor volume measurement of peripheral tumors and has been used routinely in our experiments $[9,10]$. The mice of the control group were euthanized on day 14 in accordance with the IACUC guidelines on the maximal allowed tumor load on each mouse.

\subsection{Data Analysis}

The tumor $\mathrm{pO}_{2}$ from the two LiPc implants of each tumor was measured simultaneously for 30 minutes and the data were pooled to obtain an average tumor $\mathrm{pO}_{2}$ on each day of measurements. A paired t-test was used to determine the statistical significance of the changes in $\mathrm{pO}_{2}$ within the group and an unpaired t-test was used to determine the statistical significance between groups at the same time points. The tests were two-sided, and a change with a $\mathrm{p}$ value $<0.05$ was considered statistically significant. All data are expressed as mean $\pm \mathrm{SE} ; \mathrm{n}$ is the number of animals in each group.

\section{Results}

\subsection{Effect of metronomic Cyclophosphamide on $9 \mathrm{~L}$ tumor $\mathrm{pO}_{2}$}

The baseline tumor $\mathrm{pO}_{2}$ of the CPA and the control group were $5 \pm 1 \mathrm{mmHg}$ and $8.5 \pm 2$ $\mathrm{mmHg}$ respectively and no significant difference was observed between groups, Figure 1. In the CPA treated group, a significant increase in tumor $\mathrm{pO}_{2}$ was observed from day 10 when compared with the baseline and the control group. The tumor $\mathrm{pO}_{2}$ remained at a significantly oxygenated level till day 31 and then slowly declined on day 43 after the last CPA treatment on day 21. On the other hand, no significant change in the tumor $\mathrm{pO}_{2}$ of the control group was observed during two weeks of repeated measurements.

\subsection{Effect of metronomic Cyclophosphamide on 9L tumor growth}

The tumor volumes of the CPA and the control groups were $236 \pm 45$ and $181 \pm 36 \mathrm{~mm}^{3}$ and were not significantly different, Figure 2 . A significant increase in tumor volume was observed from day 6 to day 10 in the CPA treated group as compared to the pre-treatment volume. However, the tumor volume decreased and remained at the baseline level during subsequent measurements. A significant increase in the tumor volume of the control group was observed from day 7 during 2 weeks of measurements. The tumor volume of the CPA treated group was significantly smaller than the control group on days $10-14$.

\section{Discussion}

The effect of metronomic chemotherapy is likely to vary with the dose and the interval between doses, therefore may significantly alter the therapeutic outcome. Unfortunately, a lack of appropriate marker to identify vascular changes during metronomic chemotherapy has restricted therapeutic optimization.

Our results confirm an increase in tumor $\mathrm{pO}_{2}$ during metronomic CPA therapy of 9L gliomas. The changes in tumor $\mathrm{pO}_{2}$ and tumor growth observed during these treatments are likely due to anti-angiogenic effect, which results in vessel normalization [11]. These findings support our hypothesis that tumor oxygenation will occur during metronomic chemotherapy and repeated tumor $\mathrm{pO}_{2}$ measurements can provide critical information on vessel normalization during such treatments. Tumor $\mathrm{pO}_{2}$ could be potentially used to predict outcome, identify non-responders and efficiently combine this approach with radiotherapy to achieve enhanced therapeutic outcome. EPR oximetry is successfully used to repeatedly follow the changes in tumor $\mathrm{pO}_{2}$ during metronomic chemotherapy. This technique is 
currently being tested for repeated $\mathrm{pO}_{2}$ measurements in patients with peripheral tumors undergoing chemo and/or radiotherapy. We are currently investigating the changes in microvessel density, and vascular endothelial growth factor during these treatments and how they relate to tumor $\mathrm{pO}_{2}$. Nevertheless, these results are promising and provide insight on the effect of metronomic chemotherapy on tumor oxygenation.

\section{Acknowledgments}

NIH grants CA118069 and CA120919 to NK, and P01EB2180 to HMS.

\section{References}

1. Buckner JC. Factors influencing survival in high-grade gliomas. Semin Oncol. 2003; 30:10-14. [PubMed: 14765378]

2. Buckner JC, Brown PD, O'Neill BP, et al. Central nervous system tumors. Mayo Clin Proc. 2007; 82:1271-1286. [PubMed: 17908533]

3. DeAngelis LM. Brain tumors. N Engl J Med. 2001; 344:114-123. [PubMed: 11150363]

4. Kim JT, Kim JS, Ko KW, et al. Metronomic treatment of temozolomide inhibits tumor cell growth through reduction of angiogenesis and augmentation of apoptosis in orthotopic models of gliomas. Oncol Rep. 2006; 16:33-39. [PubMed: 16786120]

5. Zhou Q, Guo P, Wang X, et al. Preclinical pharmacokinetic and pharmacodynamic evaluation of metronomic and conventional temozolomide dosing regimens. J Pharmacol Exp Ther. 2007; 321:265-275. [PubMed: 17259446]

6. Khan N, Williams BB, Hou H, et al. Repetitive tissue pO2 measurements by electron paramagnetic resonance oximetry: current status and future potential for experimental and clinical studies. Antioxid Redox Signal. 2007; 9:1169-1182. [PubMed: 17536960]

7. Khan N, Williams B, Swartz H. Clinical Applications of In Vivo EPR: Rationale and Initial Results. Applied Magn. Reson. 2006; 30:185-199.

8. Swartz HM, Clarkson RB. The measurement of oxygen in vivo using EPR techniques. Phys Med Biol. 1998; 43:1957-1975. [PubMed: 9703059]

9. Hou H, Khan N, Grinberg OY, et al. The effects of Efaproxyn (efaproxiral) on subcutaneous RIF-1 tumor oxygenation and enhancement of radiotherapy-mediated inhibition of tumor growth in mice. Radiat Res. 2007; 168:218-225. [PubMed: 17638413]

10. Hou H, Lariviere JP, Demidenko E, et al. Repeated tumor pO(2) measurements by multi-site EPR oximetry as a prognostic marker for enhanced therapeutic efficacy of fractionated radiotherapy. Radiother Oncol. 2009; 91:126-131. [PubMed: 19013657]

11. Ma J, Waxman DJ. Collaboration between hepatic and intratumoral prodrug activation in a P450 prodrug-activation gene therapy model for cancer treatment. Mol Cancer Ther. 2007; 6:2879_ 2890. [PubMed: 17989319] 


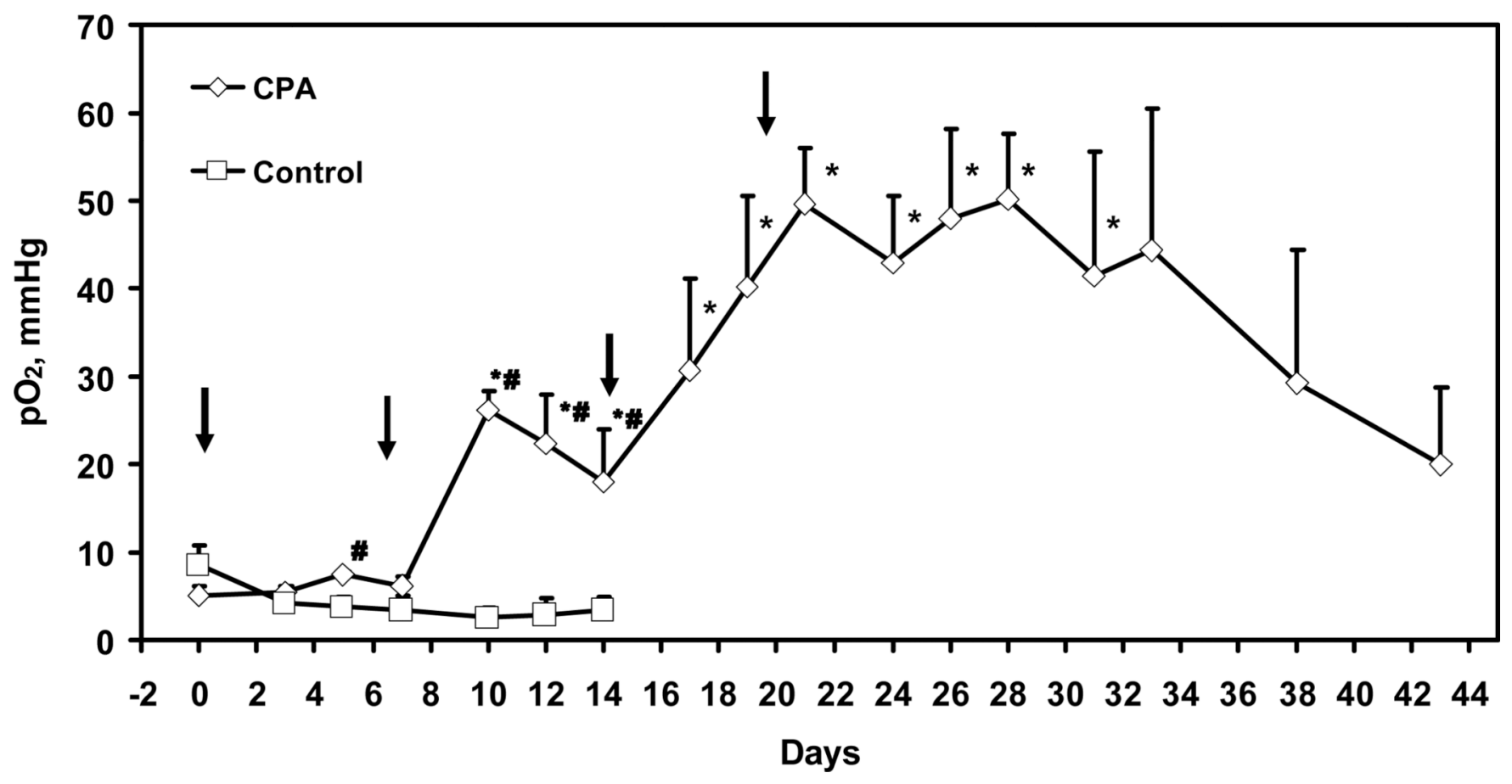

Figure 1.

Tumor $\mathrm{pO}_{2}$ of subcutaneous 9L tumors in the control $(\square)$ and CPA $(\diamond)$ treated groups. The mice were administered metronomic CPA $(140 \mathrm{mg} / \mathrm{Kg}$, i.p) on day 0 , day 7, day 14 and day 21 (indicated by arrows). Mean $+\mathrm{SE}, \mathrm{n}=3-4 .{ }^{*} \mathrm{p}<0.05$, compared with the baseline; \# $\mathrm{p}$ $<0.05$, compared with the control group. 


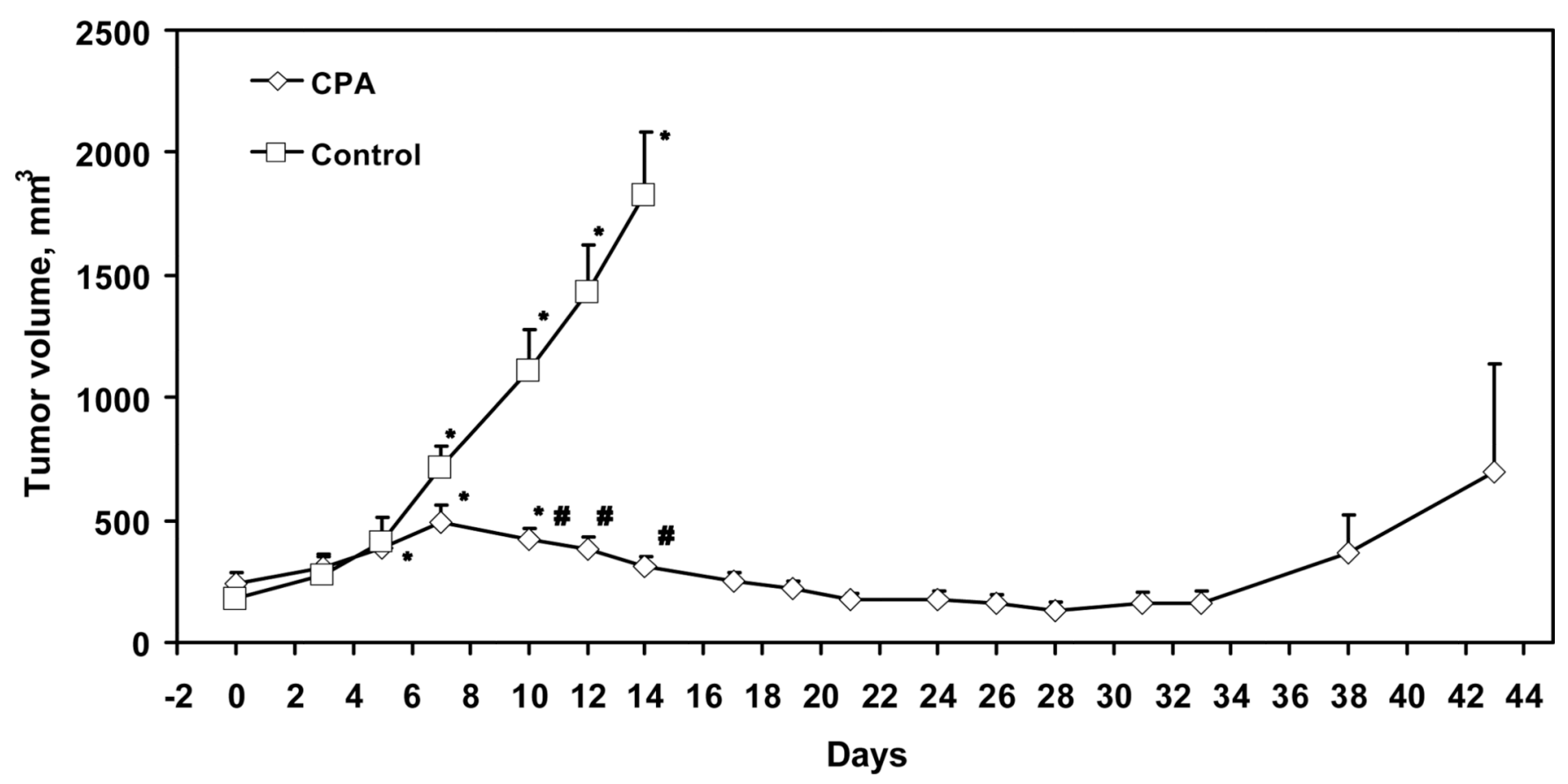

Figure 2.

The tumor volume of subcutaneous 9L tumors in the control $(\square)$ and CPA $(\diamond)$ treated groups. The mice were administered metronomic CPA (140 mg/kg, i.p) on day 0, day 7, day 14 and day 21. Mean $+\mathrm{SE}, \mathrm{n}=3-4$. ${ }^{*} \mathrm{p}<0.05$, compared with baseline; \# $\mathrm{p}<0.05$, compared with the control group. 